\title{
Study on Control Method for a Robotic Rotary Hydraulic Actuator
}

\author{
Peng Gao ' Anye Ren \\ Key Lab for Structure and Machinery, Aerospace System Engineering Shanghai, \\ Shanghai, 201109, China \\ pompon_gp@163.com
}

\begin{abstract}
Keywords: Robot, Hydraulic Servo Actuator, Control Method.
Abstract. In this work, we proposed the control method for a robotic rotary hydraulic actuator. We have designed and fabricated some prototypes of rotary hydraulic actuators. Because of valve dead zone, load disturbance and variable hydraulic pressure, the simplex PID method can't meet the control complexity. In order to improve the servo accuracy, we proposed an adaptive control method. The valve dead zone is indentified by the output feedback of the rotary actuator; the load torque is considered as the random disturbance; the good control performance and robustness are obtained by adaptive function control according to the hydraulic pressure. In the future work, we will finish a 6-Dof robot arm equipped with the rotary hydraulic joints, and further test the dynamic characteristics of the rotary hydraulic actuator.
\end{abstract}

\section{Introduction}

The hydraulic actuation has the higher power density for robotic manipulation with heavy load requirement. But compared with the electricity motor control, the hydraulic servo control are more complex, such as the hydraulic robot system work state, the external load and environment. Some hydraulic control methods have been proposed for hydraulic servo actuation.

A general mathematical function has been proposed for multivariable control of line hydraulic actuators to solve the instability problem of supply pressure variations [1]. The predictive function control with PID of a hydraulic robot system was presented with actions of random disturbance and uncertain parameters [2]. Liu et al. proposed a hybrid fuzzy PID control of hydraulic robot, where the fuzzy controller controls the piston when the piston was near the desired position, and PID controller was applied when the piston is far away the target position [3]. The explicit model reference adaptive control was also proposed to track the position of a dynamically unknown hydraulic robot [4]. A minimal control synthesis algorithm was derived to improve the servo accuracy of hydraulic quadruped robot electro-hydraulic serve actuators [5]. Sun presented the electro-hydraulic servo actuator equivalent model and a complex control strategy including robust position inner loop and impedance outer loop control [6,7]. Wang also proposed the compound control strategy including flow compensator, velocity compensator, and internal model controller [8].

Above control methods aimed at the line hydraulic actuator. In the past work, we has designed a novel rotary hydraulic actuator and fabricated some actuator prototypes. Because of the valve dead zone, load disturbance and variable hydraulic pressure, the simplex PID can't meet the control complexity. In order to improve the servo accuracy, we proposed an adaptive control method. The valve dead zone is indentified by the output feedback of the rotary actuator. The load torque is considered as the random disturbance. The good control performance and robustness are obtained by adaptive function control according to hydraulic pressure.

\section{Rotary Hydraulic Actuator}

In order to improve the output ability of conventional robotic arms, a hydraulic actuator has been designed and fabricated. Different from the general hydraulic line cylinder, the hydraulic actuator is a novel rotary hydraulic actuator with more strong power density compared with conventional motors. 

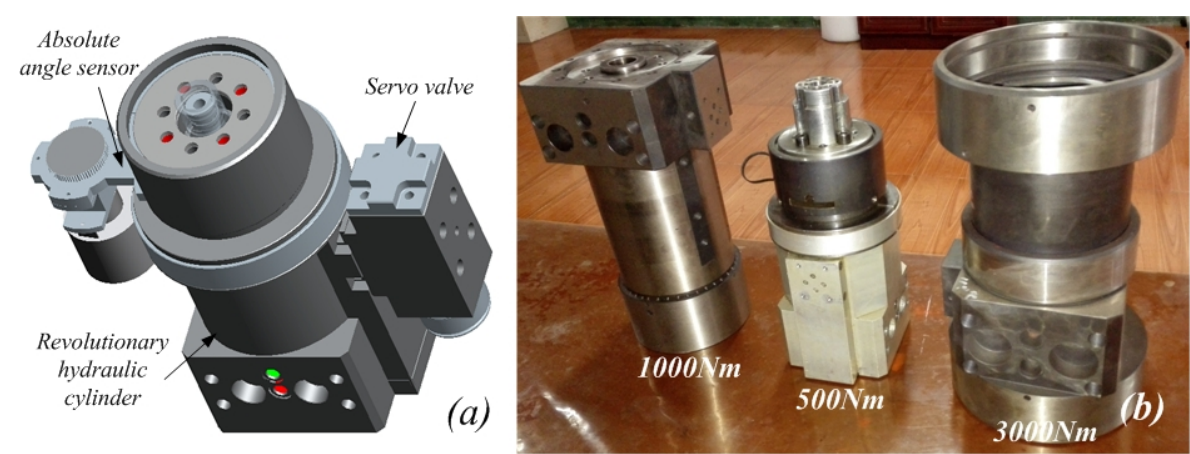

Fig. 1. Robotic rotary hydraulic actuator.

A rotary hydraulic servo system consists of a rotary hydraulic actuator, a servo valve, and an absolute angle sensor, as shown in Fig. 1(a). Since the rotary hydraulic actuator is actuated by a hydraulic pump system, the serve valve can be actuated by a small DC brushless motor with an increment angle sensor. Therefore, the closed loop control system can be established by a controller which masters the servo valve and receives the angle feedback single from the absolute angle sensor.

We have fabricated some prototypes of robotic rotary hydraulic actuators. As shown in Fig. 1(b), there are $500 \mathrm{Nm}, 1000 \mathrm{Nm}$ and $3000 \mathrm{Nm}$ actuators under $20 \mathrm{MPa}$ oil pressure, which all have the continuous rotary outputs.

\section{Hydraulic actuation analysis}

The rotary hydraulic actuator can turn the small electricity power or mechanical energy of the servo valve to the big hydraulic power and mechanical energy. The above rotary hydraulic servo system is a valve control hydraulic system. Its output characteristic presents the relation between the actuator output speed and the servo valve angle.

$$
\mathrm{v}=\frac{1}{F} \mathrm{a}\left(x_{v}-\Delta x\right) \sqrt{\frac{\mathrm{g}}{\gamma}\left(\mathrm{P}_{\mathrm{s}} \cdot \frac{f}{F}\right)}-c \frac{f}{F^{2}}
$$

where $F$ is the real cross-sectional area of the leaf blade, $x_{v}$ is the displacement of the servo valve, $\Delta x$ is the overlap of the servo valve, $P_{s}$ is hydraulic pressure, $f$ is the friction between the blade and the cylinder body.
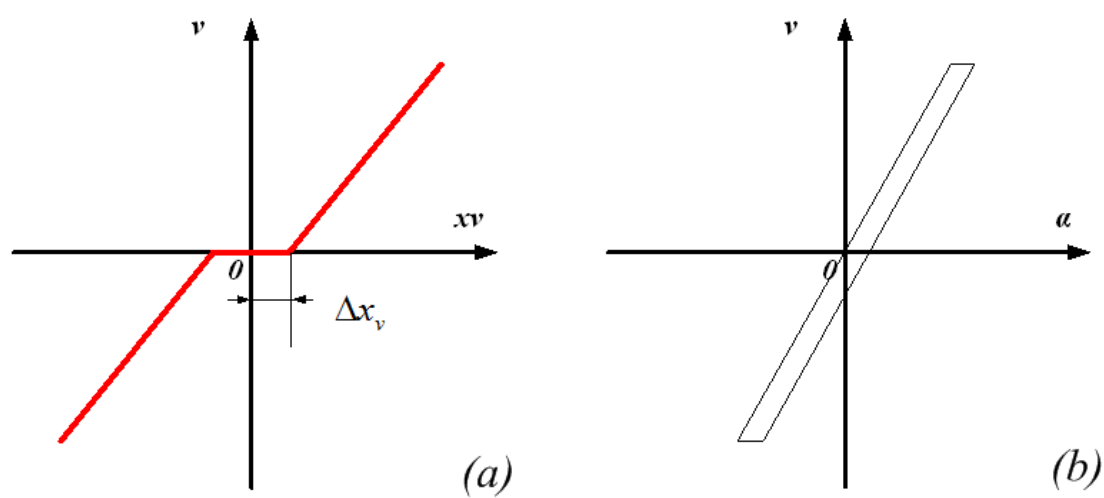

Fig. 2. Characteristic curve of the valve control system.

When $v$ is zero, the largest displacement of the servo valve is the dead zone of the valve control system. As shown in Fig. 2(a), the speed characteristic curve of the rotary hydraulic actuator presents a control dead zone of $\Delta x$, which adds the control complexity.

In addition, the servo valve is driven by a DC brushless motor with a planetary gear reducer. Because of the clearance of the reducer gear, the speed control is a hysteresis characteristic as shown in Fig. 2(b).

According to the above speed characteristic curve, we can get the speed gain $K$, hysteresis, dead zone and linearity, and other important parameters. 


$$
K=\frac{Q}{i}
$$

where $Q$ is the rate flow of the servo valve, and $i$ is the angel of the servo valve.

It is difficult to get these parameters by theoretical calculation, we mainly rely on the experiments. In order to estimate the value of the speed gain, we often use the average flow gain of the servo valve as its control gain.

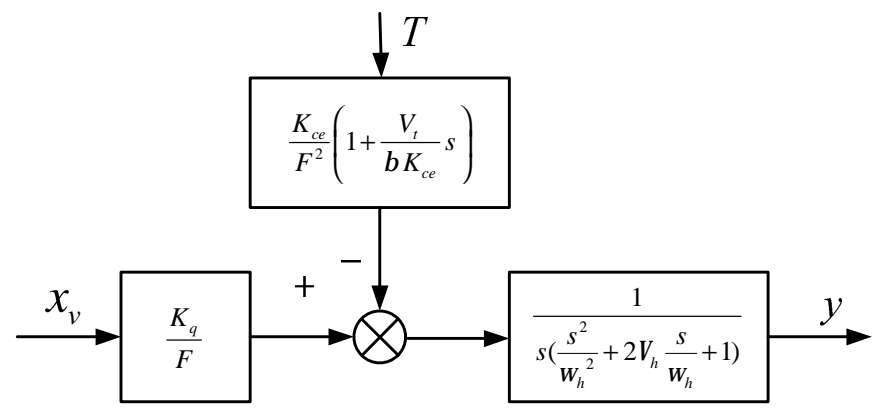

Fig. 3. Block diagram of the valve control system.

The block diagram of the valve control system is shown in Fig. 3. $T$ is the load torque, $x_{v}$ is the angle of the servo valve, and $y$ is the output angle of the rotary hydraulic actuator.

When $T$ is zero, the transfer function model between $x_{v}$ and $y$ is:

$$
\frac{Y(s)}{X_{v}(s)}=\frac{\frac{K_{q}}{F}}{s\left(\frac{s^{2}}{\omega_{h}^{2}}+2 \varsigma_{h} \frac{s}{\omega_{h}}+1\right)}
$$

In addition, the hydraulic pressure $P$ can also affect the above proportional value $K_{q}$. It is obvious that the control of the rotary hydraulic actuator is complex. The classical PID method usually can't meet the requirement of the servo control.

\section{Adaptive control}

The adaptive control method for the robotic rotary hydraulic actuator mainly includes the valve control, $T$ control and $P$ control based PID control method.

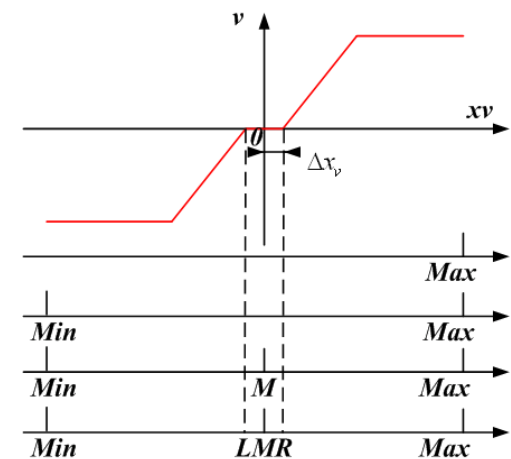

(a)

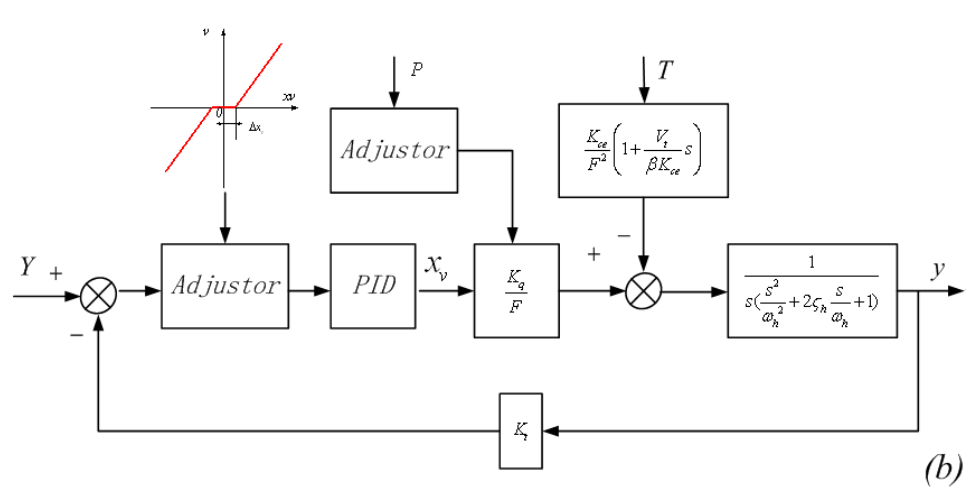

Fig. 4. Adaptive control

As shown in Fig. 4(a), after starting the control system, the hydraulic pressure is not supplied. Firstly, with no loads, the servo valve has a constant increment until the brushless motor current is more than a certain value. At this time, the angle of the servo valve is the max position. Secondly, the servo valve has a constant reduction until the brushless motor current is more than a certain value. At this time, the angle of the servo valve is the min position. Then, the hydraulic pressure is supplied, and the angle of the servo valve is controlled by identifying the output condition of the rotary hydraulic actuator until adaptively finding the dead zone. 
As shown in Fig. 4(b), in order to improve the servo accuracy of the rotary hydraulic actuator, the load torque is considered as the random disturbance. The good control performance and robustness are obtained by adaptive function control according to hydraulic pressure.

\section{Experiment}

A $1000 \mathrm{Nm}$ prototype was experimented for the servo accuracy under different hydraulic pressure.

Table 1. Results of the servo accuracy of the rotary hydraulic actuator.

\begin{tabular}{|c|c|c|c|}
\hline & $\mathbf{0}^{\circ}$ & $\mathbf{6 0}^{\circ}$ & $\mathbf{1 2 0}^{\circ}$ \\
\hline $\mathbf{5 M P a}$ & $-0.055^{\circ}$ & $-0.075^{\circ}$ & $-0.06^{\circ}$ \\
\hline $\mathbf{1 0 M P a}$ & $0.14^{\circ}$ & $-0.18^{\circ}$ & $-0.07^{\circ}$ \\
\hline $\mathbf{1 5 M P a}$ & $0.02^{\circ}$ & $-0.08^{\circ}$ & $0.1^{\circ}$ \\
\hline $\mathbf{2 0 M P a}$ & $-0.03^{\circ}$ & $-0.005^{\circ}$ & $-0.06^{\circ}$ \\
\hline
\end{tabular}

Based on the adaptive control method of valve dead zone and hydraulic pressure, the servo accuracy results are shown in Table 1 . Under $5 \mathrm{MPa}$ hydraulic pressure, the servo accuracy is controlled in $-0.075^{\circ}$. Under $10 \mathrm{MPa}$ hydraulic pressure, the servo accuracy is controlled in $-0.18^{\circ}$. Under $15 \mathrm{MPa}$ hydraulic pressure, the servo accuracy is controlled in $-0.1^{\circ}$. Under $20 \mathrm{MPa}$ hydraulic pressure, the servo accuracy is controlled in $-0.06^{\circ}$.

It can be seen that the maximum servo accuracy is less than $0.2^{\circ}$ based on the proposed prototype. Considering the resolution ratio limitation of the absolute angle sensor, the maximum servo accuracy is acceptable for the robotic applications.

\section{Conclusions}

In this work, we proposed an adaptive control method for the robotic rotary hydraulic actuator. In order to improve the servo accuracy, we proposed an adaptive control method for the valve dead zone, the load disturbance and the variable hydraulic pressure. The control method has been experimented with the good control performance and robustness. In the future work, we will finish a 6-Dof robot arm equipped with the rotary hydraulic actuators and further test its dynamic characteristic.

\section{Acknowledgements}

This work was supported by the Shanghai Science and Technology foundation (13DZ1108300).

\section{References}

[1] S. R. Habibi, R.J. Richards and A.A. Goldenberg: Hydraulic actuator analysis for industrial robot multivariable control. American Control Conference IEEE, Vol. 1(1994), p. 1003-1007.

[2] Z.H. Zhang: Predictive function cascade control scheme and its application to hydraulic robot systems. IEEE International Conference on Automation and Logistics (2009), p. 13-17.

[3] R. Liu, X. Wang, and X. Huang: Hybrid Fuzzy PID Control of Hydraulic Robot. ASME 2002 International Mechanical Engineering Congress and Exposition (2002), p. 935-938.

[4] Kireç, et al.: Experimental evaluation of a model reference adaptive control for a hydraulic robot: a case study. Robotica Vol. 21(2003), p. 71-78.

[5] J.P. Shao, et al.: Hydraulic Robot Actuator Control Based on Minimal Control Synthesis Algorithm. Journal of Sichuan University Vol. 46.6(2014), p. 178-184.

[6] G. Sun,, et al.: Hydraulic robot actuator modeling and joint angle tracking control. Yi Qi Yi Biao Xue Bao/chinese Journal of Scientific Instrument Vol. 36.3(2015), p. 584-591. 
[7] G. Sun, et al.: Hydraulic robot force control based on environment parameters adaptive estimation. Journal of Huazhong University of Science \& Technology (2015).

[8] Z. Wang, et al.: Hydraulic quadruped robot joint force control based on double internal model controller. International Journal of Control \& Automation Vol. 9(2016). 